\title{
THE VAGINAL SMEAR IN THE DIAGNOSIS OF MALIGNANCY
}

\author{
By J. Bamforth, M.D., F.R.C.P.
}

A number of years has passed since Papanicolaou and his colleagues (1943; 1948) introduced the cytological examination of the vaginal smear as an aid in the diagnosis of malignancy in the female genital tract. This method is employed extensively abroad and on an increasing scale in this country so that it should now be possible to evaluate its use in clinical practice.

The procedure should be regarded as a preliminary and additional method of diagnosis. If cells reported as positive or suspicious of malignancy are found in the vaginal smear, further investigation such as biopsy or curettage should be regarded as essential. Cytological examination might be considered unnecessary, were it not for the fact that it has brought to light many early and unsuspected cases. By 'unsuspected' cases one means those in which no biopsy would have been undertaken except for the positive report on the vaginal smear. The great majority of these cases have proved to be carcinoma of the cervix and in many no clinical suspicion of malignancy had been entertained.

There is no doubt that a high degree of accuracy in cytological diagnosis can be achieved by capable workers in the gynaecological field. Graham (1953) claimed that 90 per cent. and 70 per cent. of cancer of the cervix and cancer of the body respectively can be diagnosed by one cervical smear. Other workers have claimed similar success and Anderson and his colleagues in Edinburgh showed an accuracy of 96.4 per cent. for cervical cancer. As indicated above, the results obtained in carcinoma of the body are not so good and more false negative errors have been experienced by the majority of workers. The smear method is therefore of greater value in the diagnosis of carcinoma of the cervix. Curettage of the uterus should be performed when carcinoma of the body is suspected.

Two specimens from the vagina are usually employed for cytological examination as a routine procedure. The first is obtained by means of a pipette introduced into the posterior fornix; the second by gently scraping the surface of the cervix with a wooden spatula. The second method was intro- duced by Ayre, who devised a special form of $\stackrel{\square}{\circ}$ spatula for this purpose with a view to increasing $\vec{\omega}$ the efficiency of diagnosis of cervical carcinoma. By this means it is possible to examine a larger $\frac{C}{3}$ area of cervical tissue and also the region of the squamo-columnar junction (a common site for the $\underset{N}{\omega}$ development of cancer) more easily than by any $\omega$ other method. Nevertheless, I think that the first $\omega$ specimen is important because it contains cells which have desquamated spontaneously and it is ${ }^{\infty}$ also more suited to the diagnosis of endometrial $\frac{\circ}{2}$ cancer. In certain cases it is advantageous to $\vec{z}$ aspirate material directly from the cervical canal $\bar{z}$ and also from the endometrial cavity when carcino- $₫$ ma of the fundus or tube is suspected. Films glass slides are made from the material obtained and should be fixed immediately, whilst wet. Tis fixative commonly employed is a mixture of equi parts of 95 per cent. ethyl alcohol and ether. The are stained by Papanicolaou's solution. Alterna- $\overrightarrow{0}$ tively, the wet films can be fixed in Schaudinn's solution and stained by haemalum and eosin.

The experience gained during the last twenty years or more has shown that there are certain $\varnothing$ characteristics of malignant cells which distinguish $\overrightarrow{\overrightarrow{0}}$ them from normal cells. In the case of the more 3 typical and well differentiated neoplasms the appearances of the malignant cells may differ but? little from those of normal cells but in the greato majority of malignant growths the differences in 3 . appearance are considerable. Certain changes are $\frac{\sigma}{3}$ found both in the nucleus and in the cytoplasm. In 1934, Dudgeon and Barrett wrote that 'malig- $\frac{\delta}{2}$ nant cells stain more deeply than their benign prototypes. This is due to the fact that their nuclei 응 have a greater affinity for haemalum. They vary $D$ in size and shape; they are larger than normal and their position relative to the surrounding cytoplasm is in no way constant. The nuclearchromatin is arranged in thick, irregular and deeply $N_{0}$ staining bundles and the fine reticular arrangement ${ }_{\omega}^{N}$ of normal cells is rarely seen. The nucleoli are음 much larger than normal, they are sometimeso multiple and stand out as purple or pink dots in the sphere of the nucleus.' In addition there are $\stackrel{\mathcal{P}}{?}$ 
changes in the cytoplasm; variations in shape and size are found; aberrant and bizarre forms-e.g. Tadpole cells-may be seen. Cells showing atypical appearances may also be found in chronic inflammatory conditions, e.g. infection by Trichomonas vaginalis, and in young actively growing tissue such as a healing cervical erosion; difficulty in interpretation is usually resolved by further investigation. In a few cases cytological examination of the vaginal smear has been usefully employed in the diagnosis of such conditions as carcinoma of the ovary and of the Fallopian tube but these are exceptional. It is in the diagnosis of malignant disease of the cervix especially and of hyperplastic and carcinomatous changes in the endometrium of the body that cytology can render valuable aid and these conditions only will be dealt with in this paper.

\section{Carcinoma of the Cervix}

The great majority of cases of cancer of the cervix can be diagnosed without difficulty by clinical examination and by removal of a small piece of suspected tissue for biopsy. There is no necessity for cytological examination. Nevertheless, if cytology is to play its part in the elucidation of obscure and unsuspected cases it is important that vaginal smears should be made and sent for examination in some of the typical cases. Comparison can be made of the cell appearances seen in the smear with those found in the subsequent paraffin sections. In this way valuable experience is gained.

The importance of cytological examination lies in the fact that it leads to the discovery of many unsuspected cases of cancer, especially of the cervix. The majority of these prove to be either of the early invasive or of the intra-epithelial non-invasive type (carcinoma in situ). Graham (1953), working in Boston, recorded figures calculated on the basis of one vaginal smear. Amongst 18,303 patients in the gynaecological department examined cytologically during the period 1943 to I95I, there were 926 cases of histologically diagnosed carcinomas of the genital tract. These included 469 cases of invasive squamous cell carcinoma of the cervxi, 86 cases of intra-epithelial carcinoma of the cervix, and 206 cases of adenocarcinoma of the endometrium, of which 23,43 and 13 respectively were clinically unsuspected. Anderson and his colleagues (1953) reporting on 3,000 cases screened for cancer of the cervix in Edinburgh found 33 unsuspected cases of which 19 were of the noninvasive type and in only one was there any clinical suspicion of malignancy. More recently, Wrobel (1956) published the results obtained in a clinic of 4,200 patients in which vaginal smears were used as a screening procedure. In 18 cases histological cancer of the cervix was found. In all of them the initial clinical impression was other than carcinoma and the first positive indication was due to the cytological examination. Of these 18 cases, 10 proved to be of the early invasive and 8 of the intraepithelial type.

The following instance may be cited as an example of an unsuspected cervical carcinoma diagnosed in the first place as a result of cytological examination. I am indebted to Mr. S. G. Clayton for the clinical details.

E.P., aged 44. Married: 4-para.

7.3.55. Attended hospital complaining of postcoital bleeding for more than 8 years. Vaginal smear was taken and showed a number of atypical squamous cells with large hyperchromatic nuclei. To be repeated.

I.8.55. The second vaginal smear showed atypical cells considered to represent squamous-cell carcinoma (Figure I). The cervix was bulky with erosion on the posterior lip but was not clinically malignant.

3.8.55. Biopsy of cervix which on histological examination showed carcinoma in situ (Figure 2).

10.8.55. Total hysterectomy with bilateral salpingo-oophorectomy and removal of cuff of vagina. The cervix was completely examined by division into several pieces. Sections showed several foci of carcinoma and one small focus with early invasion.

The term 'dyskaryosis' is used by Papanicolaou (1954) to denote certain early malignant changes in the cell which are limited almost entirely to the nucleus. This may show enlargement, irregularity, hyperchromia and multinucleation. The cytoplasm may show vacuolation but the cell as a whole retains its original type as contrasted with the aberrant forms seen in advanced malignancy. Papanicolaou considered that dyskaryosis occurs in the intra-epithelial stage or more rarely with early invasion. Wied (1956), amplifying this conception, described certain criteria in the examination of the smear for the differentiation of non-invasive and invasive cancer. I do not think that the cytological changes can indicate with certainty yet whether it is a case of invasive or non-invasive carcinoma ; in order to decide this question one must have recourse to biopsy. In any case this is essential to confirm the cytological findings. Herein difficulty may arise. In some cases a suspicious area or areas may be seen but in others the clinical appearances are such that it may be impossible to decide where to take a biopsy. In addition, as already seen in the case described above, the lesion may be multifocal. Some gynaecologists employ a circular biopsy or conisation of the cervix to overcome this difficulty. The examination of the tissue removed may reveal an invasive growth but in some cases only an intraepithelial lesion may be found. Even after serial 


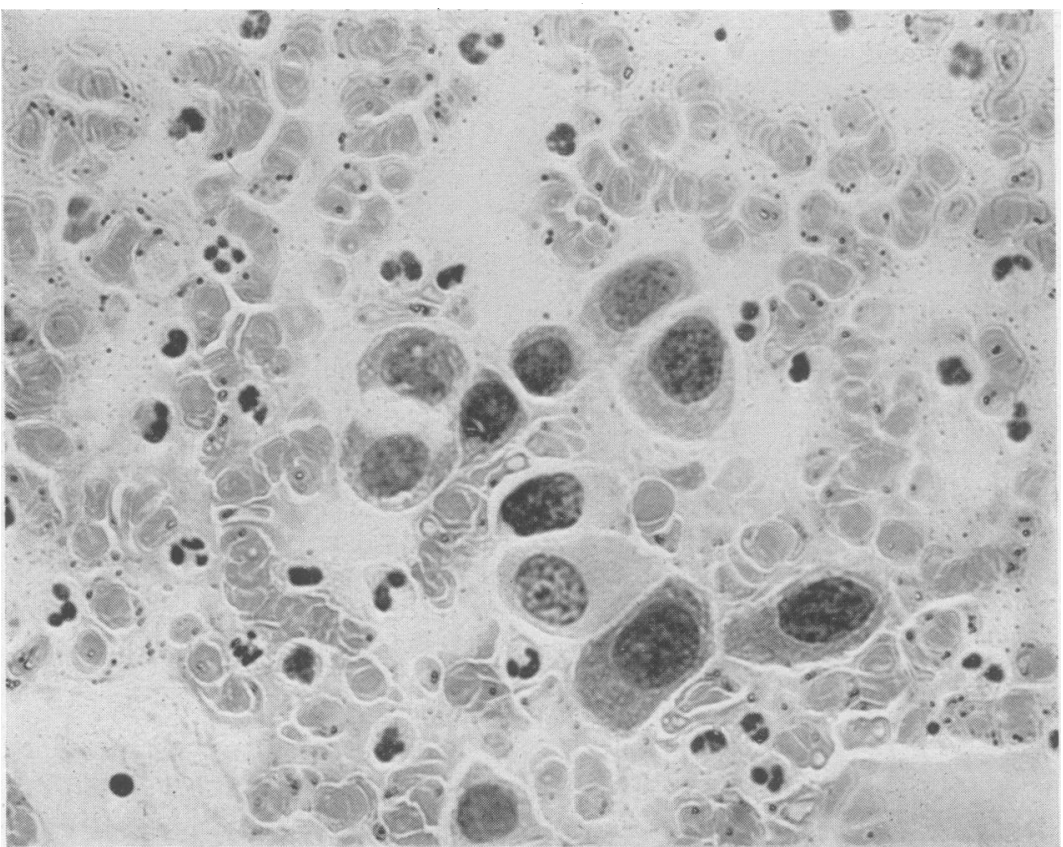

FiG. I.-Smear showing malignant cells of squamous type. $\times 550$. Haemalum and eosin.

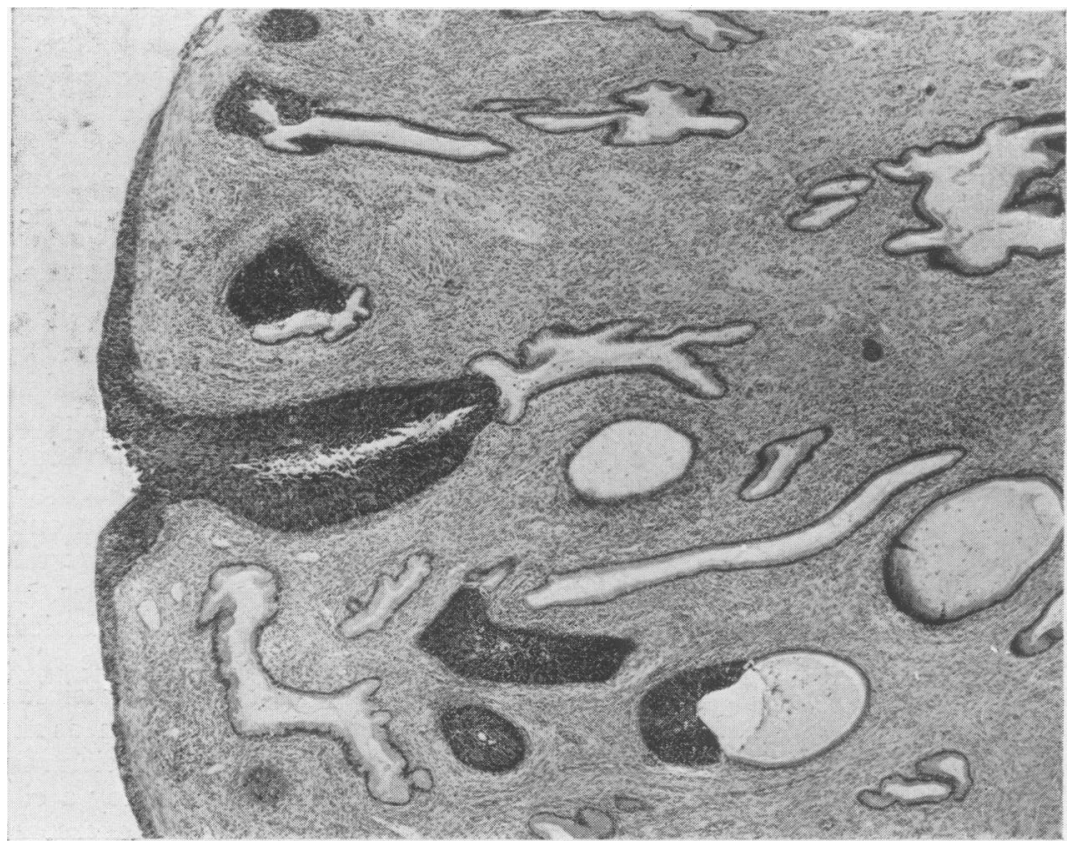

Fig. 2.-Biopsy from case shown in Fig. I. Carcinoma in situ. Extension into the glands is not regarded as invasive. $\times 145$. Haemalum and eosin. 


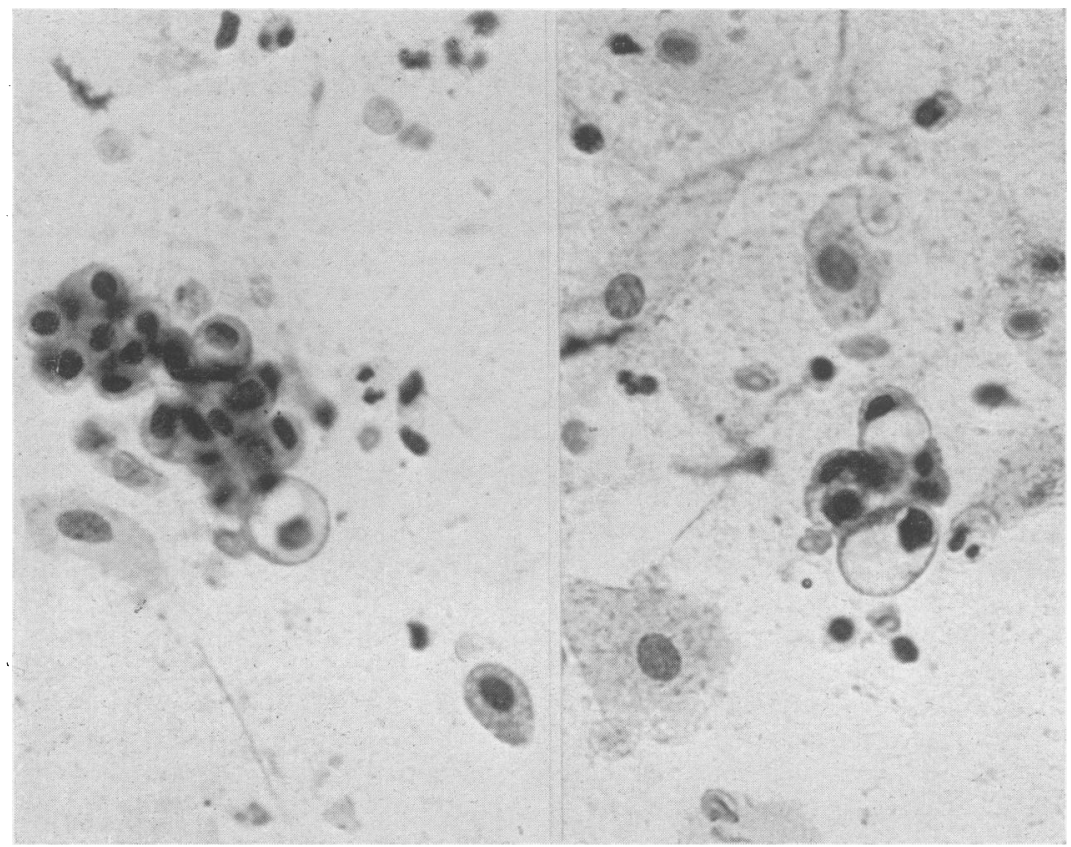

FIG. 3.-Vaginal smear showing two clumps of malignant cells in a case of carcinoma of the endometrium: diagnosis confirmed histologically. $\times 550$. Haemalum and eosin.

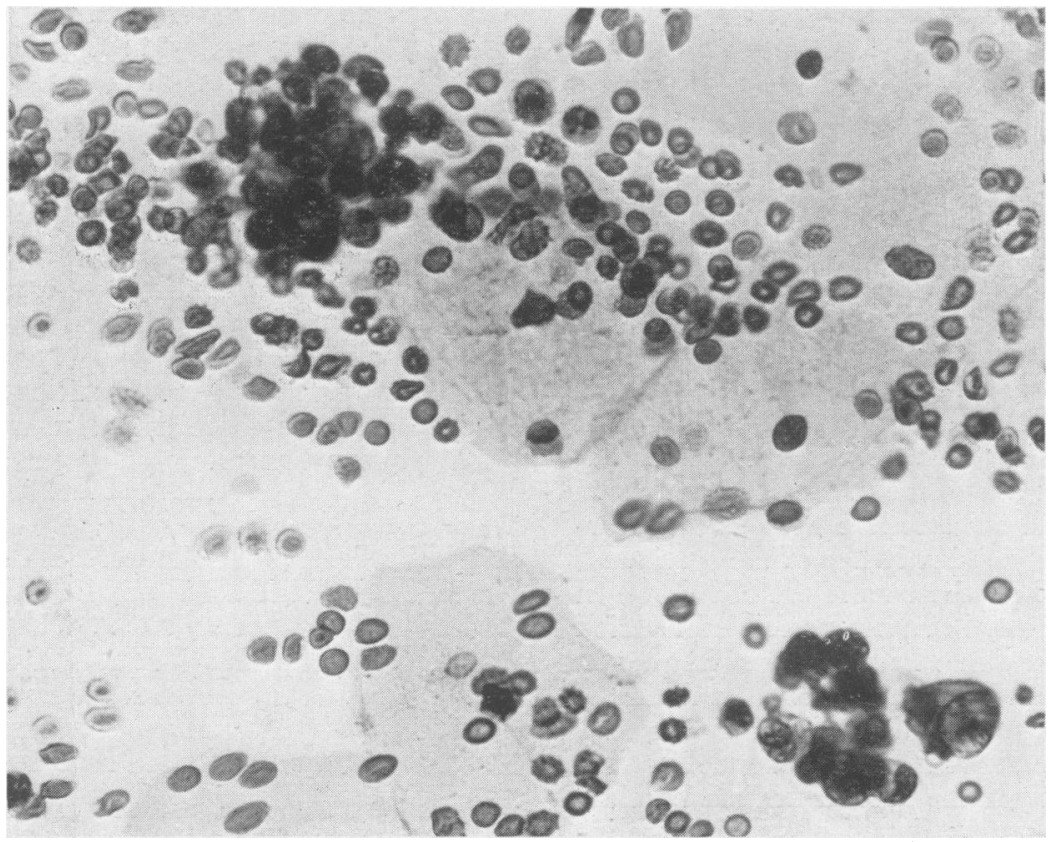

Fig. 4.-Vaginal smear showing two clumps of endometrial cells. Histological diagnosis-cystic hyperplasia of the endometrium. $\times 550$. Haemalum and eosin. 
sections the question of invasion may be doubtful; such cases have been described as borderline.

It appears to be impossible to forecast what may happen to any given case of intra-epithelial carcinoma of the cervix. More information is still needed on this subject which constitutes an important problem in gynaecology to-day. There is no doubt that a certain number of these cases pass into typical invasive cancer, sometimes after many years have elapsed. Recently, however, increasing evidence has been presented to show that some which have been carefully followed-up without treatment for a varying period have shown complete regression. A few of such cases have occurred in pregnant women. That pre-invasive carcinomatous lesions in the cervix may occur in pregnancy as distinct from epithelial changes resulting from pregnancy has been shown-by Greene and his fellow-workers (1953-54). There appears to be no demonstrable histological difference between the lesions which regress and those which persist. The result has been that some gynaecologists during recent years have preferred, especially in younger patients, to adopt a conservative and watching attitude instead of the more radical methods of treatment. It is here that cytology can play a useful part as one would naturally expect. It is known that the vaginal smear is extremely accurate in detecting in its earliest stages, and before it can be recognized clinically, local recurrence after operation for invasive carcinoma of the cervix.

\section{Carcinoma and Hyperplasia of the Endo- metrium}

In carcinoma of the body of the uterus the appearance of a blood-stained discharge usually arouses the early attention of the patient and unsuspected cases are not so likely to be discovered by the vaginal smear as in the case of the cervix; nevertheless, a certain number are found. In the large series of cases reported by Graham (mentioned above) there was 13 unsuspected carcinomas of the body. It is somewhat surprising that Wachtel and Plester (1952) found seven unsuspected cases of carcinoma of the uterus in smears from $r, 853$ patients and of these five were cases of carcinoma of the body and only two of the cervix.

In clinical practice the case of the older women in the pre-menopausal or post-menopausal period suffering from uterine haemorrhage frequently presents a problem in diagnosis. Apart from the question of malignancy, the vaginal smear may give useful information in the assessment of ovarian function, especially in the post-menopausal period. The following cases, for details of which I am indebted to Mr. A. J. Wrigley, are illustrative :

M.M., aged 55, married. Menopause 9 years ago.

Admitted complaining of intermittent brownish vaginal discharge for 9 months.
Examination of the vaginal smear showed few superficial epithelial cells but a fair number of $\frac{a}{\zeta}$ intermediate and basal cells. Many red cells and $\stackrel{\mathbb{Q}}{\varrho}$ leucocytes. Small clumps of malignant cells in-.. dicating adenocarcinoma of papilliferous type $\overrightarrow{\vec{F}}$ were found (Figure 3 ) and this was confirmed by $\stackrel{\infty}{+}$ histological examination of curettings from the body of the uterus.

This case may be contrasted with the following : $\frac{\bar{D}}{\bar{D}}$

B. C., aged 73, married. Menopause 33 years ago. $\stackrel{\square}{\square}$

Admitted complaining of post-menopausal hae- क morrhage for one month.

Examination of the vaginal smear showed large numbers of superficial epithelial cells; red cells $\overrightarrow{\vec{\omega}}$ ++ ; polymorphonuclear leucocytes, scanty.

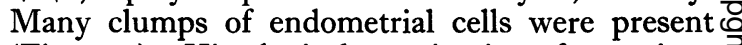
(Figure 4). Histological examination of curettings 3 revealed a condition of cystic and stromal hyperplasia of the endometrium.

Endometrial cells are found in the vaginal smear $\omega$ at the time of menstruation but also, sometimes of in large numbers, in metropathia haemorrhagica associated with hyperplasia of the endometrium. 을 In this condition, due to the marked oestrogen effect, there is a thickened vaginal epithelium and $\bar{O}$

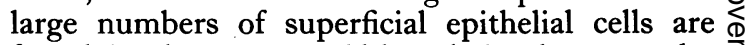
found in the smear. Although in the examples given above the distinction is clear, in some case it is difficult to distinguish the clumps of endome्ष $\overrightarrow{0}$ trial cells found in hyperplasia from the malignain clumps in adenocarcinoma. Endometrial cers tend to be more uniform in shape and size. Leucocytes are frequently associated with adenocarcinoma and are found ingested by the malignant cells but in hyperplasia the leucocytes, when present, are found in between the endometrial $\stackrel{\mathbb{D}}{\varrho}$ cells.

In conclusion it would appear that the vaginal 3 smear has established itself as a valuable mode of examination in gynaecological practice. Without having recourse to its wholesale employment in the female population, I feel that it could be used with advantage on a more extensive scale in gynaeco- $\frac{0}{3}$ logical departments.

\section{BIBLIOGRAPHY}

ANDERSON, A. F., GRANT, M. P. S., McBRYDE, R. M., and COCKBURN, M. K. (1953), $\ngtr$. Obstet. Gynaec., Brit. Emp., 응 60, 345 .

AYRE, J. E. (1947), Amer. F. Obstet. Gynec., 53, 609.

DUDGEON, L. S., and BARRET'T, N. R. (1934), Brit. F. Surg., 을 22, 4

GRAHAM, R. M. (1953), F. Obstet. Gynaec., Brit. Emp., 60, 483.

GREENE, R. R., PECKHAM, B. M., CHUNG, J. T., BAYLY, o M. A., BENARON, H. B. W., CARROW, L. A., and N GARDNER, G. H. (1953), Surg., Gynec., Obstet., 96, 71.
PANICOLAOU, G. N. (1954), 'Atlas of Exfoliative Cytology,' N PAPANICOLAOU, G. N. (1954), 'Atlas of Exfoliative Cytology,' $\omega$

PAPANICOLAOU, G. N., and MARCHETTI, L. A. (1948), 'The Epithelia of Woman's Reproductive Organs,' New York.

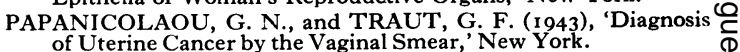

WACHTEL, E., and PLESTER, J. A. (1952), f. Obstet. Gynaec., Brit. Emp., 59, 323.

WIED, G. L. (1956), Amer. F. Obstet. Gynec., 71, 793.

WROBEL, C. J. (1956), Ibid., 7I, 402. 